\title{
An Overview of Products and Bias in Research
}

\author{
David Gloss ${ }^{1}$
}

Published online: 23 July 2015

(C) The American Society for Experimental NeuroTherapeutics, Inc. 2015

\begin{abstract}
Cannabis is a genus of annual flowering plant. Cannabis is often divided into 3 species-Cannabis sativa, Cannabis indica, and Cannabis ruderalis - but there is significant disagreement about this, and some consider them subspecies of the same parent species. Cannabis sativa can grow to 5-18 feet or more, and often has a few branches. Cannabis indica typically grows 2-4 feet tall and is compactly branched. Cannabis ruderalis contains very low levels of $\Delta-9$ tetrahyocannabinol so is rarely grown by itself. Cannabis ruderalis flowers as a result of age, not light conditions, which is called autoflowering. It is principally used in hybrids, to enable the hybrid to have the autoflowering property. There are $>700$ strains of cannabis, often with colorful names. Some are strains of 1 of the 3 subspecies. Many are crossbred hybrids. The strains can be named in a variety of ways: smell or lineage are common ways of naming. There are only a few rules about how the strains are named, and most strains' names do not follow the rules. There are 4 basic preparations of marijuana: bhang, hasish, oil (or hash oil), and leaves and/ or buds. In medical marijuana trials, subjective outcomes are frequently used but blind breaking can introduce significant bias. Blind breaking occurs when patients figure out if they are in the control or the treatment group. When this occurs, there is significant overestimation of treatment effect.
\end{abstract}

Keywords Cannabis $\cdot$ C. sativa $\cdot$ Bias $\cdot$ Medical marijuana

David Gloss

davy.gloss@gmail.com

1 Department of Neurology, Geisinger Clinic, 100 N Academy Ave, Danville, PA 17822, USA

\section{Cannabis Plant Information}

Cannabis is a genus of annual flowering plants [1]. It is dioecious, meaning that some plants have male flowers and others have female flowers. Some plants have both male and female flowers. It is wind pollinated. The leaves are palmate, meaning that they form leaflets which radiate from the base of the leaf. The leaflets are serrated [2]. The plant is indigenous to Asia. Cannabis can be identified from similar plants by looking at some of its characteristics [1].

Cannabis plants have small hairs called trichomes. There are several different types of trichomes [3]. The trichomes on female plants are particularly high in $\Delta$-9-tetrahyocannabinol (THC) content. The purpose of the trichomes is not entirely clear, but may be, in part, to trap insects that would try to eat the plant.

Cannabis belongs to the family Cannabaceae [1]. The family Cannabaceae is composed of flowering plants, and includes hops plants and a type of tree called hackberries, as well as cannabis. The main similarities among the members of this family typically include palmate leaves, dioecious flowers, and wind pollination.

Cannabis is often divided into 3 species-Cannabis sativa, Cannabis indica, and Cannabis ruderalis - but there is significant disagreement about this [4-6]. There are also some named varieties, for example Cannabis indica var. kafristanica, and Cannibis sativa var. spontanea [4]. Each species can interbreed with the other species, leading to hybridization. One of the disagreements is whether this should be one species or different species. One definition of species is one in which a group of organisms can interbreed in nature. Using this definition, some consider cannabis a single species of $C$. sativa L. (Linnaeus) with subspecies sativa, indica and ruderalis [1,7]. Others, disagree, arguing that the capacity for hybridization should not be 
the major determinant for some plants, but rather phytochemical composition, leading to the idea that they should be considered different species [4].

Cannabis sativa is the most commonly occurring subspecies in the West. It is a tall thin-leaved plant, which flowers under certain light conditions. Flowering is initiated when darkness exceeds $11 \mathrm{~h}$ per day [1]. It can grow to 5-18 feet or more, and often has a few branches.

Cannabis indica is more broad-leaved than $C$. sativa. It is most commonly shorter and has more leaves and buds, giving it a more bushy appearance. The buds tend to be wider. It typically grows 2-4 feet tall, and is compactly branched.

Cannabis ruderalis may have originated from southern Russia. It contains very low THC, so is rarely grown by itself. It has the property of autoflowering: flowers appear as a result of plant age rather than light conditions. It is principally used in hybrids, to enable the hybrid to have the autoflowering property. The plants are smaller than $C$. sativa and have adapted to colder temperatures than $C$. sativa. It does not typically grow to be more than 2 feet tall and is unbranched [2].

It is important to note that there is ongoing hybridization, to create new strains and select desirable characteristics. One of the characteristics frequently chosen is THC content. Between 2000 and 2004, the average THC content of marijuana plants from the Netherlands rose from $8 \%$ to $20 \%$ [8]. A larger multinational view also saw an increase over time, but not as strong as seen in the Netherlands [9].

While there is some concern with regard to the high THC content of modern hybrids, there is probably no added therapeutic effectiveness. In one study among medical marijuana users in the Netherlands, 3 different preparations of pharmaceutical-grade cultivated cannabis, which have been tested and verified for their components, were compared. The preparations contained $19 \%$ THC, $<1 \%$ cannabidiol (CBD); $12 \% \mathrm{THC},<1 \% \mathrm{CBD}$; or $6 \% \mathrm{THC}$, $7.5 \%$ CBD. Patients were asked to rate 12 adjectives on a scale of 1 to 100 , to describe the subjective experiences. They did not find significant differences, except that increased appetite was seen in both the $19 \%$ and $12 \%$ THC preparations $(p=0.03)$; the feelings of dejection and anxiety were both higher in the high THC group than in the low THC group ( $p=0.006$ and $p=0.004$, respectively) [10]. Another study looked at patients with HIV/AIDS, and compared $C$. sativa, $C$. indica, and 2 hybrids. They found significant differences in only in two areas. Cannabis indica had increased energy (mean difference of 1.53) and appetite (mean difference of 1.79) compared with $C$. sativa and a smaller difference to other marijuana on a visual analogue scale of 1 to 10 [11].

In an Australian study looking at various constituents of marijuana, the content of total THC varied between $1 \%$ and $40 \%$ in samples tested. CBD varied between $0 \%$ and $6 \%$ [12].

\section{Strains}

There are $>700$ strains of recreational cannabis, most with colorful names [13]. In some states, the recreational forms of cannabis can be used for medical purposes. Some are strains of 1 of the 3 subspecies. Most are crossbred hybrids. The strains can be named in a variety of ways. Some names are based on odor; others are predicated on lineage. There are only a few rules about how the strains are names, and most names do not follow the rules.

There are a couple examples where there does seem to be some sort of naming convention. Kush typically indicates either pure $C$. indica or a $C$. indica hybrid. Afghan Kush, Hindu Kush, Green Kush, and Purple Kush are all pure C. indica strains. Blueberry Kush and Golden Jamaican Kush are hybrids based on C. indica. For $C$. sativa, there are similar examples using the words Diesel and Haze, with some strains being pure $C$. sativa, and some strains being hybrids of C. sativa. Diesel is named for its smell.

\section{Marijuana Preparations}

There are 4 basic preparations of marijuana: bhang, hasish, oil (or hash oil), and leaves and/or buds [1].

Bhang is fresh leaves and flowers that have been ground into a paste with a mortar and pestle. Bhang is used in a drink made with spices called Bhagi Ki Thandai, or made into balls called bhang golis. Bhang is used in India, Bangledesh, and other countries as part of religious festivals. It is particularly featured as part of the festival of Holi. Holi is the festival of color, or love, and is well known for the day when people can throw colored water or powder at each other.

Hashish is composed of trichomes from the cannabis plant, extracted in various ways. Its THC content is somewhat variable, and depends on the method of creation, as well as the source plants, but reportedly can increase the amount of THC by up to 8 times [1].

Oil (or hash oil) is taking marijuana and using solvents to extract the oil containing THC. If butane is the solvent, it is called dabs. The potency of oil is thought to be up to $90 \%$ THC [14]. Users of dabs report that there is more tolerance [average increase of 0.68 on a 4-point scale $(p<0.001)$ ] and withdrawal [average increase of 0.22 on a 4-point scale $(p<0.001)]$ than with flower preparations [15].

Leaves and/or flowers/flower buds are ingested or smoked. Some smoke them with tobacco, which can be called a blunt.

\section{Pharmacologic Preparations}

Nabiximols (Sativex; GWPharma, Cambridge, UK) is an oromucosal spray [16]. Each spray contains $2.7 \mathrm{mg}$ THC 
and $2.5 \mathrm{mg}$ CBD. There have been randomized, controlled studies using nabiximols. It has been used in studies of the treatment of multiple sclerosis (MS)-related spasticity, neuropathic pain, and bladder complaints. It has also been used in studies considering its role in the treatment of neuropathic and cancer pain [17-19]. Nabiximols are indicated in the UK for the treatment of moderate-to-severe spasticity related to MS, which has not responded adequately to other antispasticity medication. In Canada, the indications are more broad: treatment of spasticity and neuropathic pain in MS, and as adjunctive pain relief for patients with advanced cancer. It is not approved in the USA. Patients may use up to 12 sprays per day, although in open-label treatment some patients use more. The spray bottle is made so that after priming, one 10-ml bottle delivers 90 sprays.

Nabilone (Cesamet; MEDA Pharmaceuticals, Somerset, NJ, USA) is a synthetic cannabinoid made to mimic THC [20]. It is indicated for use in nausea and vomiting associated with chemotherapy, in patients who have failed to respond adequately to conventional antiemetic treatments. It is a schedule II drug in the USA. Dosing is 1-2 mg twice daily, with a maximum of $2 \mathrm{mg} 3$ times daily. Side effects are a significant concern with this medication, with very frequent reporting of vertigo, dizziness, and/or dry mouth. Many patients treated will experience disturbing psychotomimetic reactions not observed with other antiemetic agents, although some smaller trials report much lower numbers of patients being affected. This may be due to statistical variation, differences in titration, or differences in patient characteristics.

Dronabinol (Marinol; AbbVie, North Chicago, IL, USA) is a synthetic form of THC [21]. It is indicated by the Food and Drug Association for use in anorexia-associated weight loss in adult patients with AIDS, and in nausea and vomiting associated with cancer chemotherapy in patients who have failed to respond adequately to conventional antiemetic treatments. It is a schedule III drug in the USA. If used for appetite stimulation, the dose is $2.5 \mathrm{mg}$ before lunch and dinner, although there is significant variation in the dosing needed. If used for nausea and vomiting, $5 \mathrm{mg} 3$ or 4 times a day works for most patients. Notably, dronabinol and prochloroperazine taken together seem to improve efficacy.

\section{Issues with Medical Marijuana Trials}

There are many different systems for measuring the risk of bias in studies. One of the systems used extensively in neurology is the system used in the journal Neurology [22]. The system uses an ordinal I-IV grading of papers, where Class I is a perfect randomized, controlled trial (RCT); II is a flawed RCT; III is a seriously flawed RCT or an observational trial; and IV is expert opinion, case reports, or other trials with so high a risk of bias to be no better than expert opinion. In this system, many papers are classified as Class IV. Observational trials, in this system, may very rarely qualify for Class II, but generally qualify for Class III or IV.

A recent systematic review of medical marijuana was intended to be a guideline but was turned into a systematic review because there was a paucity of high quality evidence [23]. In this review, nearly half of the studies were rated as Class IV. Of the remaining half, one quarter were rated as Class III. The most common reasons for studies being downgraded are as follows: $<80 \%$ of patients completing the study; baseline characteristics being presented and either substantially equivalent or adjustment for differences; and a lack of outcome assessment which was objective, masked, or performed by someone outside the treatment team. This paper used the previous version of the process manual [24].

In the newer version of the process manual, there were significant changes to the grading system [22]. The most significant change is that a paper is deemed Class IV if it does not have a comparison group of some sort. Other important changes included making it harder to qualify for Class I. Class I papers need to include allocation concealment (the investigators are prohibited from being able to manipulate which treatment arm the patient is allocated to). Noninferiority trials have their own special criteria in order to qualify as Class I.

A nice example of how the grading can impact the creation of community standards can be seen in the 4 randomized trials of epilepsy [25]. These were all small trials, which used CBD alone, and had a combined number of patients enrolled of $<$ 60. In either the newer or older system, all 4 of these papers would be graded as Class IV. As such, in the Neurology review, no recommendation could be made with regard to epilepsy [23].

The prohibition against subjective outcome assessments is particularly problematic in medical marijuana trials. According to the systematic review, some of the best evidence for medical marijuana comes from trials of spasticity in MS. There is a consensus that objective measure like the Ashworth scale should not be used but that subjective measures should be used. Therefore, some trials should and are using subjective measures, but this can bring about other sources of bias.

One area where using subjective outcome measures is particularly worrisome is with blind breaking. Even with highquality trials, individual patients may believe they understand their treatment allocation. This can occur owing to the adverse effects of the drug, as well as previous experience with recreational marijuana. With marijuana, and cannabinoids, it is a commonly held belief that many patients will know their treatment allocation. If there is a subjective outcome, as it typical for many trials using marijuana, blind breaking can introduce significant bias.

There was an important examination of the effect of blind breaking combining the patient data from 3 randomized, placebo controlled trials of nabiximols, which included 
subjective outcome measures [26]. They compared patients who had previously used cannabis, so would be more likely to break the blinding, with patients without such previous use. They did not find any difference in efficacy, or in side effects between the 2 patient groups. As this is the typical way that blind breaking is tested, it seems that this may not be such a difficult problem for cannabis researchers.

Required Author Forms Disclosure forms provided by the author are available with the online version of this article.

\section{References}

1. United Nations Office on Drugs and Crime. Recommended methods for identification and analysis of cannabis and cannabis products. United Nations, New York, 2009.

2. Anderson LC. Leaf variation among cannabis species from a controlled garden. Botanical Museum Leaflets Harvard Univ 1980;28;61-69.

3. Potter DJ. The propagation, characterisation and optimisation of Cannabis sativa L. as a phytopharmaceutical. $\mathrm{PhD}$ thesis, King's College, London, 2009.

4. Hillig KW and Mahlberg PG. A chemotaxonomic analysis of cannabinoid variation in Cannabis (Cannabaceae). Am J Botany 2004;91:966-975.

5. Russo EB. History of cannabis and its preparations in saga, science, and sobriquet. Chem Biodivers 2007;4:1614-1648.

6. Erkelens JL, Hazenkamp. That which we call Indica, by any other name would smell as sweet. Cannabinoids 2014;9:9-15.

7. Small $\mathrm{E}$ and Cronquist A. A practical and natural taxonomy for cannabis. Taxon 1976;25:405-435.

8. Pijlman FTA, Righter SM, Hoek, J, Goldschmidt HMJ, Niesink RJM. Strong increase in total delta-THC in cannabis preparations sold in Dutch coffee shops. Addict Biol 2005;10:171-180.

9. Cascini F, Aiello C, Di Tanna GL. Increased delta-9tetrahydrocannabinol (delta-9-THC) content in herbal cannabis over time: systematic review and meta-analysis. Drug Abuse Rev 2012;5:32-40.

10. Brunt TM, van Genugten M, Honer-Snoeken K, van de Velde M J, Nieseink RJM. Therapeutic satisfaction and subjective effects of different strains of pharmaceutical-grade cannabis. J Clin Psychopharmacol 2014;34:344-349.

11. Corral VL. Differential effects of medical marijuana based on strain and route of administration: A 3-year observational study. $J$ Cannabis Ther 2001;1:43-59.
12. Swift W, Wong A, Li KM, Arnold JC, McGregor IS. Analysis of cannabis seizures in NSW, Australia: Cannabis potency and cannabinoid profile. PLoS ONE 2013;8:e70052.

13. Snoeijer W. A checklist of some Cannabaceae cultivars. Part 1: Cannabis. Div. Pharmacognosy, Leiden/Amsterdam Centre for Drug Research, Leiden, the Netherlands, 2001.

14. Ghosh TS, Van Dyke M, Meffey A, Whitley E, Erpelding D, Wolk L. Medical marijuana's public health lessons-Implications in retail marijuana in Colorado. N Engl J Med 2015;372:993-995.

15. Loflin M, Earleywine M. A new method of cannabis ingestion: The danger of dabs? Addict Behav 2014;39:1430-1433.

16. SATIVEX (nabiximols). Package insert. GW Pharma Ltd, Salisbury, UK (revised December 2014).

17. Nurmikko TJ, Serpell MG, Hoggart B, et al. Sativex successfully treats neuropathic pain characterized by allodynia: A randomized, double-blind, placebo-controlled trial. Pain 2007;133:210220.

18. Johnson JR, Burnell-Nugent M, Lossignol D, Ganae-Motan ED, Potts R, Fallon MT. Multicenter, double-blind, randomized, placebo-controlled, parallel-group study of the efficacy, safety, and tolerability of THC;CBD extract and THC extract in patients with intractable cancer-related pain. J Pain Symptom Manage 2010;39: 167-179.

19. Johnson JR, Lossignol D, Burnell-Nugent M, Fallon MT. An open-label extension study to investigate the long-term safety and tolerability of THC/CBD oromucosal spray and oromucosal THC spray in patients with terminal cancer-related pain refractory to strong opioid analgesics. J Pain Symptom Manage 2012;46:207-218

20. CESAMET (nabilone). Package insert. Meda Pharmaceuticals, Quebec, Canada (revised September 2013).

21. MARINOL (dronabinol). Package insert. Solvay Pharmaceuticals, Marietta, GA (revised September 2004).

22. American Academy of Neurology. Clinical practice guideline process manual, $2011 \mathrm{Ed}$. The American Academy of Neurology, St. Paul, MN, USA.

23. Koppel BS, Brust JC, Fife T, et al. Systematic review: Efficacy and safety of marijuana in selected neurologic disorders. Report of the Guideline Development Subcommittee of the American Academy of Neurology. Neurology 2014;82:1556-1563.

24. American Academy of Neurology. Clinical practice guideline process manual, 2004 Ed. The American Academy of Neurology, St. Paul, MN, USA.

25. Gloss D, Vickrey B. Cannabinoids for epilepsy. Cochrane Database Syst Rev 2014;3:CD009270.

26. Wright S, Duncombe P, Altman DG. Assessment of blinding to treatment allocation in studies of cannabis-based medicine $\left(\right.$ Sativex $\left.{ }^{\mathbb{R}}\right)$ in people with multiple sclerosis: a new approach. Trials 2012;13:189. 